\title{
The Incidence and Risk of Inducing Hyperthyroidism Following Amiodarone Treatment
}

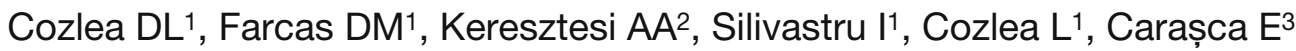 \\ ${ }^{1} 3^{\text {rd }}$ Medical Clinic, University of Medicine and Pharmacy, Tîrgu Mureș, Romania \\ 2 Institute of Legal Medicine, University of Medicine and Pharmacy, Tîrgu Mureș, Romania \\ $34^{\text {th }}$ Medical Clinic, University of Medicine and Pharmacy, Tîrgu Mureș, Romania
}

\begin{abstract}
Introduction: Amiodarone, a frequently used antiarrhythmic drug in cardiology, is very efficient in the treatment of ventricular and supraventricular tachiarrithmyas. The iodine content of amiodarone is 39\%. Its chemical structure is similar to tyrosine. It is estimated that $1-23 \%$ of patients treated with amiodarone can develop hyperthyroidism. The purpose of this study is to assess and monitor the incidence of hyperthyroidism induced by amiodarone in patients admitted for various types of cardiac dysrhythmias, considering that most of the patients included in the study came from an endemic goitre area.

Material and method: One-hundred patients chosen systematically (62 men, 38 women) from 560 patients treated with amiodarone in the January 1st, 2009 - December 31, 2009 period were assessed (clinically, laboratory findings and imaging studies); their mean age was 64 years (range 50-70 years). In order to identify and diagnose hyperthyroidism, a questionnaire (Newcastle index) was used, also urinary iodine dosage, hormonal dosages (T3, T4, TSH) and thyroid imaging (ultrasound, radioactive iodine uptake test) were used.

Results: The incidence of hyperthyroidism induced by amiodarone in this study was $8 \%$. Hyperthyroidism was more frequent in women $(6$ women vs. 2 men).

Conclusions: Thyroid hormonal levels have to be determined and a clinical or thyroid ultrasound examination should be made prior to initiating a treatment with amiodarone. At the end of the study, 8 patients, most of them female, were diagnosed with hyperthyroidism.
\end{abstract}

Keywords: hyperthyroidism, amiodarone, Newcastle index

Received: 9 April 2013

\section{Introduction}

Iodine is indispensible for thyroid hormonogenesis. The quantity of iodine in the body is one of the most important factors in thyroid function adjustment. It is estimated that $1-23 \%$ of patients treated with amiodarone can develop hyperthyroidism $[1,2]$.

Two physiopatological mechanisms of hyperthyroidism correlated with an increased intake of iodine have been observed:

- aggravated hyperthyroidism due to iodine excess in patients with thyroid pathology, and

- iodine induced hyperthyroidism that occurs in case of patients without known thyroid pathology $[2,3]$.

Due to isotope dilution there is a low iodine capture level in all iodine charges, but in patients with existing thyroid pathology, iodine capture is not as low as previous research shows $[4,5,6]$.

The diagnosis of hyperthyroidism through iodine overcharge is suggested by the patient's history: iodine agents used in practice or used in investigations [7]. The diagnosis can be confirmed by total iodine dosage or by iodine measurement. The iodine charge is confirmed when iodine blood levels exceed $400 \mu \mathrm{g} / 24$ hours and the measurement is useful since it confirms the patient's history, as well as the suspicion of thyrotoxicosis due to an increased intake [8].

Correspondence to: Arthur Atilla Keresztesi

E-mail: ar2r_a23@yahoo.com
Thyroid hormone dosage is important because amiodarone diminishes the metabolic clearance of T4 (thyroxine) and the peripheral conversion of T4 in T3 (triiodothyronine) and increases the total and free $\mathrm{T} 4$ concentration, and the T3 concentration remains normal or lowers. The diagnosis of hyperthyroidism is based on the low circulating TSH (thyroid stimulating hormone) levels and on the absence of its response after stimulation with TRH (thyroid releasing hormone) $[9,10,11]$.

The purpose of this study is to assess and monitor the incidence of hyperthyroidism induced by amiodarone in patients admitted for various types of cardiac dysrhythmias, considering that the patients included in the study came from an endemic goitre area.

\section{Material and method}

The study was approved by the Ethics Committee of the University of Medicine and Pharmacy of Tîrgu Mures, Romania, and was performed in the accordance with sthe tandards of the Declaration of Helsinki. Written consent for using the patient's data in the present study was obtained from all patients. No procedures beyond the standard of care were performed specifically for the purposes of this study.

For this study, 560 patients met the inclusion criteria: all of them were treated with amiodarone at the site of the study, for a period of 6 months for different cardiac dysfunctions: dilatative cardiomiopathy, angina pectoris, 


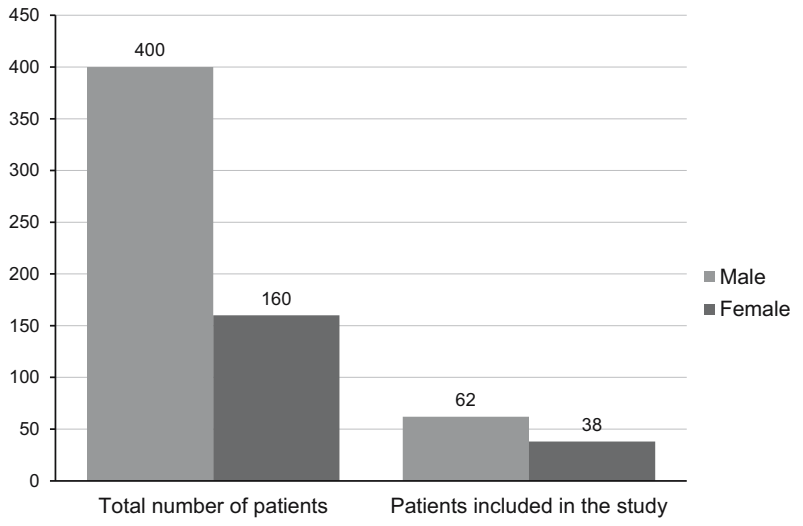

Fig. 1. Distribution of patients by gender

atrial flutter with variable blockage, sinus node dysfunction, chronic atrial fibrillation (AF) with medium ventricular rate, paroxysmal AF with high ventricular rate, ventricular premature beats (ventricular extrasystoles), sustained ventricular tachiarrithmyas, acute myocardial infarction.

The group of 560 patients was hospitalized between January 1st, 2009 - December 31, 2009. The patients were inserted in a database, in the order of their hospitalization date and every $5^{\text {th }}$ or $6^{\text {th }}$ patient was chosen. At the end of the process, 100 patients were systematically chosen from the group and included in the study (Figure 1). Some of the patients were from another county, and their progress could not be thoroughly followed, therefore in some cases the $6^{\text {th }}$ and not the $5^{\text {th }}$ patient was included in the study. For simpler data analysis and statistical calculus, only 100 patients were chosen.

The Newcastle index was applied to all 100 patients to determine the clinical grounds for hyperthyroidism diagnosis. For scores smaller than 10 the thyroid function is considered to be normal, for scores between 11 and 19 there is a possible hyperthyroidism, while for scores over 20 the diagnosis of hyperthyroidism is certain.

After the Newcastle index was calculated for all patients, those with possible hyperthyroidism were subjected to a series of blood tests to determine the level of thyroid hor-

Table I. Newcastle index for diagnosing hyperthyroidism - subjective symptoms

\begin{tabular}{lcc}
\hline Subjective symptoms & Yes & No \\
\hline Dyspnoea on effort & 1 & 0 \\
Palpitations & 2 & 0 \\
Fatigue & 2 & 0 \\
Preference for heat & 0 & -5 \\
Preference for cold & 5 & 0 \\
Excessive sweating & 3 & 0 \\
Nervousness & 2 & 0 \\
Increased appetite & 3 & 0 \\
Decreased appetite & 0 & -3 \\
Gain of weight & 0 & -3 \\
Loss of weight & 3 & 0 \\
\hline
\end{tabular}

mones TSH, T3 and T4, thyroid ultrasound, radioactive iodine uptake test (RAIU test), cholesterol levels, achilean reflexograme, iodine urine levels (rapid urinary iodide test kit by Merck KGaA, Darmstadt, Germany).

\section{Results}

Out of the 100 patients treated with amiodarone, 62 were male and 38 female. The average age of these 100 patients was 64 years, with ages ranging from 50 to 70 years.

The interpretation of Newcastle index results, regarding hyperthyroidism diagnosis, revealed the following:

- 78 patients had no specific symptoms for hyperthyroidism (Newcastle score under 10);

- 18 patients presented clinical signs and symptoms for hyperthyroidism (Newcastle score between 11 and 19);

- 4 patients had Newcastle scores higher than 20.

For further assessment of the Newcastle's score sensitivity, clinical investigations were carried out for the $22 \mathrm{pa}$ tients suspected of thyroid dysfunction (with a score over 10). The results confirmed $8(36.4 \%)$ patients with hyperthyroidism, 6 female and 2 male.

Out of the 8 patients with confirmed thyroid dysfunction, 4 had high Newcastle score and 4 had a medium Newcastle score (11-19).

\section{Discussions}

In the present study, the incidence of amiodarone induced hyperthyroidism was $8 \%$, all patients were over 50 years old, the average age being 64 years. Thyroid dysfunction was more frequent in women. An important aspect to mention is that all patients came from areas with iodine deficiency. A similar incidence of thyroid dysfunction induced by amiodarone had been found in another Romanian study [13]. The $8 \%$ incidence found in the present study is representative for the Transylvania region, this being a known goitre endemic area. It is worth mentioning that recently there have not been other studies carried out in this area.

Although the Newcastle index indicated that $22 \mathrm{pa}$ tients might have hyperthyroidism, only 8 were confirmed.

Table II. Newcastle index for diagnosing hyperthyroidism - objective signs

\begin{tabular}{lcc}
\hline Objective signs & Present & Absent \\
\hline Palpable thyroid & 3 & -3 \\
Bruit over thyroid & 2 & -2 \\
Exophthalmos & 2 & -2 \\
Lid retraction & 2 & 0 \\
Lid lag & 1 & 0 \\
Hyperkinesis & 4 & -2 \\
Finger tremor & 1 & 0 \\
Hot hands & 2 & -2 \\
Uneven pulse & 4 & 0 \\
Moist hands & 1 & -1 \\
\hline
\end{tabular}


$100 \%$ of those with high score were confirmed, thus, for this group of patients the Newcastle index has been reliable, but in case of medium scores (11-19), the Newcastle index has proved to be irrelevant in the present study $(22 \%$ had confirmed hyperthyroidism).

A smaller incidence of amiodarone induced hyperthyroidism of only 3\% has been noted in a study conducted by Hoffman et al in 2008 [12]. While the male:female ratio in the aforementioned study was 3:1, in our study it was 1:3. In both studies, most patients were older than 55 years.

Radioactive iodine uptake test proved to be of assistance in diagnosing a thyroid adenoma in 4 patients. Iodine capture was low or absent in 4 other cases, but correlated with the clinical symptoms of hyperthyroidism, this being characteristic for thyroid dysfunction induced by excess iodine. In determining the etiology of the hyperthyroidism, thyroid ultrasound did not prove to be of assistance, except for diagnosing hyperechogenic nodules. Hormone dosage was an essential method to confirm the suspicion of hyperthyroidism: T3, T4 with values above normal and low TSH.

Thyroid dysfunction was reported between 3 and 6 months after initiating amiodarone treatment. A possible explanation of this delay is that prior to initiating amiodarone treatment, no investigations concerning the thyroid function were carried out. The treatment of the thyroid dysfunction consisted in amiodarone stoppage and administration of betablockers. In a study conducted by Lambert et al [14] on 24 patients, it has been found that after 1-4 months of amiodarone treatment, serum T4 levels incresead by an average of $40 \%$ above the pretreatment levels, in some cases the elapsed time between the first symptoms and paraclinical modifications being shorter than in our study.

\section{Conclusions}

1. The incidence of hyperthyroidism in this study was $8 \%$. All patients came from areas with iodine deficiency.
2. Women were more often affected than men and in all patients with high Newcastle index diagnosis of hyperthyroidism was clinically confirmed.

3. An important practical aspect to keep in mind before initiating amiodarone treatment is at least to determine the thyroid hormone level, to conduct a clinical examination and thyroid ultrasound, due to the amiodarone's increased iodine content which may lead to hyperthyroidism.

\section{References}

1. Martino E, Bartaleta L, Bogazzi $F$ et al. The effects of amiodarone on the thyroid. Endocrine Rev. 2001;22:240-254.

2. Davies TF, Larsen PR. Thyrotoxicosis. In: Larsen, Kronenberg, Melmed, Polonsky "Williams Textbook of Endocrinology", 10 th Ed. Saunders, 2003;411-421.

3. Franklyn JA, Sheppard MC. Amiodarone and thyroid dysfunction. Trends Endocrinology Metab. 1993;4:128-131.

4. Becker KL. Principles and practice of endocrinology and metabolism, 3rd J.B Lippincott Williams and Wilkins Company, Philadelphia. 2001;24-56.

5. Bogazzi F, Bartalena L, Brogioni S et al. Color flow Doppler sonography rapidly differentiates type I and type II amiodarone-induced thyrotoxicosis Thyroid. 1997:7:541-545.

6. Brien JF, Jimmo S, Brennan FJ et al. Distribution of amiodarone and its metabolite, desethylamiodarone, in human tissues. Can J Physiol Pharmacol 1987;65(3):360-364.

7. Wiersinga WM. Amiodarone and the thyroid. In: Weetman AP, Grossman A eds. Pharmacotherapeutics of the thyroid gland. Berlin: Springer. 1996; 225-287.

8. Sundick RS, Herdegeden DM, Brown TR et al. The incorporation of dietary iodine into thyroglobuline increases its immunogenicity. Endocrinology. 1987;120(5):2078-2082.

9. Chiovato L, Martino E, Tonacchera M et al. Studies on the in vitro cytotoxic effect of amiodarone. Endocrinology, 1994;134(5):2277-2282.

10. Goodman and Gilman's. The pharmacological basis of therapeutics, The McGraw-Hill Companies. 9th edition. 1996;103-110.

11. Guyetant S, Rousselet MC, Wion-Barbot N et al. Hyperthyroidism induced by amiodarone and hyperthyroidism induced by iodine. Histologic, immunohistochemical and ultrastructural aspects. Anne Pathol. 1995;15 (6):431-437.

12. Hofmann A, Nawara C, Ofluoglu S et al. Incidence and predictability of amiodarone-induced thyrotoxicosis and hypothyroidism. Wien Klin Wochenschr. 2008;120(15-16):493-498.

13. Trifanescu R, Fica S, Barbu C et al. Amiodarone-induced thyroid dysfunction in cardiac patients from areas with iodine deficiency. 2004;42(3):595-605.

14. Lambert MJ, Burger AG, Galeazzi RL et al. Are selective increases in serum thyroxine (T4) due to iodinated inhibitors of T4 monodeiodination indicative of hyperthyroidism? J Clin Endocrinol Metab. 1982;55:1058-1065. 